\title{
Splash Flow from a Metal Plate Hit by an Electron Beam Pulse
}

\author{
M. Garcia
}

September 4, 1997

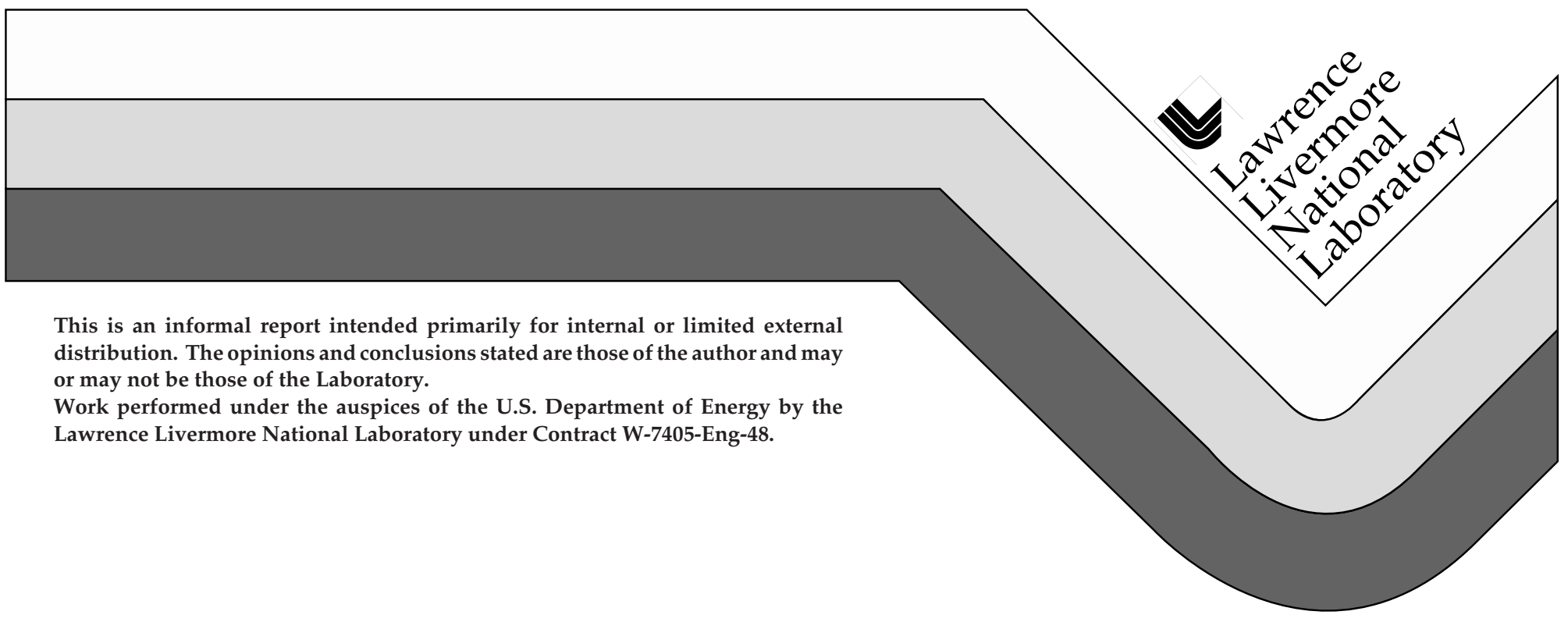




\section{DISCLAIMER}

This document was prepared as an account of work sponsored by an agency of the United States Government. Neither the United States Government nor the University of California nor any of their employees, makes any warranty, express or implied, or assumes any legal liability or responsibility for the accuracy, completeness, or usefulness of any information, apparatus, product, or process disclosed, or represents that its use would not infringe privately owned rights. Reference herein to any specific commercial product, process, or service by trade name, trademark, manufacturer, or otherwise, does not necessarily constitute or imply its endorsement, recommendation, or favoring by the United States Government or the University of California. The views and opinions of authors expressed herein do not necessarily state or reflect those of the United States Government or the University of California, and shall not be used for advertising or product endorsement purposes.

This report has been reproduced directly from the best available copy.

Available to DOE and DOE contractors from the Office of Scientific and Technical Information P.O. Box 62, Oak Ridge, TN 37831

Prices available from (615) 576-8401, FTS 626-8401

Available to the public from the National Technical Information Service

U.S. Department of Commerce 5285 Port Royal Rd. Springfield, VA 22161 


\title{
Splash flow from a metal plate hit by an electron beam pulse
}

\author{
Manuel Garcia \\ 4 September 1997
}

Lawrence Livermore National Laboratory, L-153, POB 808, Livermore, CA 94550 garcia22@1lnl.gov, (510) 422-6017, FAX (510) 423-5080

When a pulsed electron beam hits a metal plate with sufficient energy a volume of the metal becomes hot fluid that subsequently sprays out of the plate. A second pulse of electrons traveling toward the plate would scatter and degrade before impacting the solid plate because of its encounter with the diffuse material of the initial splash. People designing electron beam machines for use as pulsed radiation sources wish to eliminate the interaction between the electrons and the splash because they want sharp radiation pulses emitted from the solid plate. This report presents a compressible fluid model of this splash flow and compares specific cases with experiments and comprehensive calculations performed by B. DeVolder and others at the Los Alamos National Laboratory, see reference (1).

My aim was to develop as simple a theory as possible to calculate the speed and density of the splash flow. I have used both simplifying assumptions and mathematical approximations to develop convenient formulas. As I wished to make a clear and interesting presentation of this work to a diverse audience that includes people outside the specialty of fluid dynamics, some of my descriptions may seem wordier than necessary.

The plan of the report is as follows. In the section called "energy deposition" I describe how an electron beam deposits energy in a solid plate, converting some of the material into a hot fluid. The initial temperature of this fluid is the key parameter in determining the nature of the subsequent flow; an explicit formula is shown. Flow occurs in two regions: along a streamtube within the metal plate and as an expanding plume outside the metal plate. Flow within the plate is described in the section called "isentropic flow." This flow occurs as 
expansion waves move at the speed of sound through the streamtube. The analysis of this flow provides a formula for the mass flow over time from the plate into the external splash. The section called "centered expansion" elaborates on the nature of certain approximations I have made in treating the wave phenomena in both the streamtube and splash flows. The section called "splash flow" presents a formula to describe the material density as a function of space and time outside the plate. This formula depends on the timedependent material density at the plate, which was found during the streamtube analysis. The section called "examples" shows the results of specific calculations and a comparison to computational and experimental results described in reference (1). The final section, "possible future work," poses new questions.

\section{Energy deposition}

Electrons of kinetic energy $\mathrm{E}$, in $\mathrm{MeV}$, travel a distance $\lambda$, in centimeters, upon entering solid matter,

$$
\lambda=(0.542 \cdot E-0.133) / \rho
$$

where $\rho$ is the mass density in grams per cubic centimeter. This formula is an approximation for all materials and for electron energy above $0.8 \mathrm{MeV}$, see Fermi (2). Individual electrons scatter within the target material as they travel, losing energy and diffusing laterally from the original direction of the electron beam. The motion of the electrons is isotropic by the time they reach the range depth $\lambda$. The volume of material absorbing electron energy has a trumpet shape with an inlet radius $r_{0}$ equal to the electron beam radius. The volume I chose is a trumpet with a radius of

$$
r(x)=\lambda\left[\left(1+\frac{\mathrm{r}_{0}}{\lambda}\right)-\sqrt{1-\frac{X}{\lambda}}\right]
$$

where $x$ is the distance into the target material. This trumpet has an initial half angle of $26.6^{\circ}$ and a terminal angle of $90^{\circ}$ at $x=\lambda$. I chose this shape as a compromise between a conical volume of depth $\lambda$ and a trumpet with a quarter circle profile and of equal depth. All three volumes have a base radius of $\lambda+r_{0}$. I judged the cone to have too rapid a diffusion of electrons with depth, and the quarter circle 
trumpet to have too slow a diffusion. The volume for any depth up to $x=\lambda, V(x)$, is $\pi$ times the integral over $x$ of the radius squared.

Electrons with kinetic energy between 1 and $20 \mathrm{MeV}$ deposit an average of $(1 / \rho) \mathrm{dE} / \mathrm{dx}=1.6 \mathrm{MeV} \cdot \mathrm{cm}^{2} / \mathrm{gm}$ for all materials. This approximation is based primarily on the data for "restricted stopping power" of helium shown by Berger and Seltzer (3). The energy in MeV deposited by an electron in its transit through a plate which is thinner than a range depth is the product of density, thickness, and the constant 1.6. A pulse containing $\mathrm{N}_{\mathrm{e}}$ electrons deposits $(1.6 \times$ $\left.10^{6}\right) \cdot \rho \cdot \Delta \mathrm{L} \cdot \mathrm{N}_{\mathrm{e}} \mathrm{eV}$ of energy into a volume of depth $\Delta \mathrm{L}$. The total number of atoms in this volume is $\rho \cdot \mathrm{N}_{\mathrm{A}} \cdot V(\Delta \mathrm{L}) / \mathrm{A}_{\mathrm{W}}$, with $\mathrm{N}_{\mathrm{A}}$ being Avogadro's Number and $A_{w}$ being the atomic weight. The ratio of $\mathrm{eV}$ deposited per atom is $\left[\left(1.6 \times 10^{6}\right) \cdot \rho \cdot \Delta \mathrm{L} \cdot \mathrm{N}_{\mathrm{e}}\right] /\left[\rho \cdot \mathrm{N}_{\mathrm{A}} \cdot V(\Delta \mathrm{L}) / \mathrm{A}_{\mathrm{W}}\right]$. Notice that the energy of the electron beam influences this ratio through the volume function.

The trumpet-shaped volume of material absorbing the electron energy is assumed to become a perfect gas at temperature T. For a perfect gas,

$$
\begin{aligned}
& \Delta h=\mathrm{C}_{\mathrm{p}} \cdot \Delta T \\
& \mathrm{C}_{\mathrm{p}}-\mathrm{C}_{\mathrm{V}}=\mathrm{R}=\frac{\mathrm{k}}{\mathrm{m}} \\
& \frac{\mathrm{C}_{\mathrm{p}}}{\mathrm{C}_{\mathrm{V}}}=\gamma \\
& \mathrm{C}_{\mathrm{p}}=\frac{\gamma}{\gamma-1} \cdot \frac{\mathrm{k}}{\mathrm{m}}
\end{aligned}
$$

where $\Delta h$ is enthalpy change, $\Delta T$ is temperature change, $\mathrm{c}_{\mathrm{p}}$ and $\mathrm{c}_{\mathrm{v}}$ are the specific heats at constant pressure and volume, respectively, $\mathrm{R}$ is the gas constant, $\mathrm{k}$ is Boltzmann's constant, $\mathrm{m}$ is the atomic mass, and $\gamma$ is the ratio of specific heats. The temperature of the fluid in $\mathrm{eV}$ is given by $(\gamma-1) / \gamma$ times the enthalpy-mass product, shown earlier as the ratio of eV deposited per atom, 


$$
\mathrm{T}=\frac{\gamma-1}{\gamma} \cdot \frac{\left(1.6 \times 10^{6}\right) \cdot \rho \cdot \Delta \mathrm{L} \cdot \mathrm{N}_{\mathrm{e}} \cdot \mathrm{A}_{\mathrm{W}}}{\rho \cdot \mathrm{N}_{\mathrm{A}} \cdot V(\Delta \mathrm{L})}
$$

The $\gamma$ for a monatomic gas is $5 / 3$.

\section{Isentropic flow}

The fluid volume is established instantly in comparison to the travel time of stress waves through the plate material. Also, the duration of the electron beam pulse is assumed to be shorter than the travel time of waves across the plate. For example, assuming a $1.0 \mathrm{~mm}$ thick plate and a (stress or shock) wave speed of $1.0 \mathrm{~cm} / \mu \mathrm{s}$ then an electron beam pulse shorter than 100 ns would appear as an impulse. The fluid is assumed to be a single component atomic gas because any initial ionization would quickly recombine within the solid density fluid.

If the plate is thicker than the range depth of the electrons then the fluid flows out of the back side of the plate and toward any subsequent electron beam pulses. If the plate is thinner than the range depth of the electrons then fluid flows both backward and forward. Backward flow issues from an area $\pi r_{0}{ }^{2}$, while forward flow issues from an area $\pi r(\Delta \mathrm{L})^{2}$. The flow within the plate is treated as a one-dimensional, isentropic expansion of a perfect gas. The onedimensional approximation is very reasonable for plates thin compared to the electron range depth (only slight flare for the streamtube), yet with a thickness larger than the electron beam diameter (streamtube length/diameter $\geq 1$ ). I used Shapiro (4) for a description of compressible flow.

At time $t=0$ a streamtube of fluid, $V(\Delta \mathrm{L})$, is created at temperature $\mathrm{T}$, with a total mass $\mathrm{M}_{0}$, and density $\rho_{0}$, what is the subsequent flow? The region outside the plate is assumed to be a vacuum. Consider a streamtube that is open at both ends. An expansion wave is launched from each exit area into the streamtube and the fluid behind each wave flows toward the respective exit. Fluid that has yet to sense a wave remains static. Eventually the two oppositely directed expansion waves meet at the midpoint, fluid everywhere is now in motion. After the two waves cross at the 
midpoint, the fluid between them is now "behind" both waves and the oppositely directed accelerations cancel, leaving a region of static fluid that grows from the midpoint out toward the exits. The entire volume is static when the waves reach the opposite ends of the streamtube. The expansion waves travel at the sound speed, $\mathrm{c}=\sqrt{ }[\gamma \mathrm{eT} / \mathrm{m}]$, for $\mathrm{T}$ in $\mathrm{eV}$, so they traverse the streamtube in a time $\Delta \mathrm{L} / \mathrm{c}$. The mass flow at each exit area is choked (maximum flow per unit area, "Fliegner's formula"), it is at Mach number $M=1$. The total mass flow out of the tube during the period $0 \leq t \leq \Delta \mathrm{L} / \mathrm{c}$ is

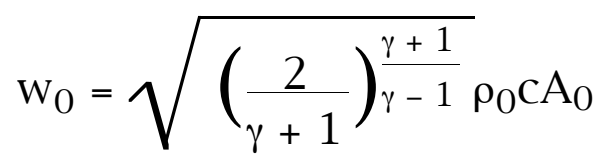

where $A_{0}$ is the sum of the two exit areas. The total mass, $M_{1}$, and density, $\rho_{1}$, at time $\mathrm{t}_{1}=\Delta \mathrm{L} / \mathrm{C}$ are

$$
\begin{aligned}
& \mathrm{M}_{1}=\mathrm{M}_{0}-\mathrm{w}_{0} \mathrm{t}_{1} \\
& \rho_{1}=\frac{\mathrm{M}_{1}}{V(\Delta \mathrm{L})}
\end{aligned}
$$

where the subscript is an index of the number of wave traversals. With isentropic flow there are no heat losses, so the temperature of static fluid at time $t_{1}$ is unchanged (stagnation temperature). In using an isentropic flow description I assume that heat flows out of the plate with the fluid rather than being lost by conduction, radiation, or dissipative effects (irreversibly).

The process described above occurs again during the time interval $\Delta \mathrm{L} / \mathrm{c} \leq t \leq 2 \Delta \mathrm{L} / \mathrm{c}$, and with the starting values $\mathrm{T}, \mathrm{M}_{1}, \rho_{1}$, and $\mathrm{w}_{1}$ (like $\mathrm{w}_{0}$ but with $\rho_{1}$ in place of $\rho_{0}$ ). In this way the mass flow into the vacuum $\left(\mathrm{w}_{0}, \mathrm{w}_{1}, \mathrm{w}_{2}, \ldots\right)$ decreases in the manner of a temporal staircase as sound waves bounce back and forth through the fluid volume in the plate. A streamtube closed at one end behaves exactly like one half of the double-ended streamtube described. 


\section{Centered expansion}

The expansion waves described above are idealized as zerothickness, constant-velocity "clumped waves," with finite property changes across them. In reality they are a sequence of wavelets with infinitesimal property changes across each, which as a group form a wave of finite thickness with a wave head that moves at higher speed than the tail. The initial wavelet launched at $t=0$ travels into undisturbed fluid at temperature $\mathrm{T}$, and on experiencing the wavelet the fluid cools and accelerates toward the launch point. Motion lowers the fluid pressure, however unless this pressure now matches the exit area boundary condition, here $p=0$, then a subsequent wavelet is launched. This second wavelet enters fluid at lower temperature $\mathrm{T}-$ $\Delta \mathrm{T}$, so it moves at a slower sound speed $\mathrm{c}=\sqrt{ }[\gamma \mathrm{e}(\mathrm{T}-\Delta \mathrm{T}) / \mathrm{m}]$. Other wavelets follow in a similar manner. On an $x$-t plot of the onedimensional isentropic flow, a clumped wave appears as a line with slope $1 / \mathrm{c}$ and the finite thickness wave appears as a fan emanating from the launch site $x=0$ (and $x=\Delta \mathrm{L}$ with negative slope). The clumped wave approximation collapses the incrementalism of the fan onto the initial wavelet. Figure 1 is an $x-t$ schematic of clumped and finite expansion waves, fluid particles paths are shown as dotted lines.

A centered expansion is the two-dimensional acceleration of a steady flow about some pivot point, usually a corner, or the onedimensional impulsive exhausting of a pressurized tube into a vacuum. The term "centered expansion" describes the fan-like appearance of the sequence of expansion wavelets which either emanate from a corner in the $x-y$ space of two-dimensional steady flow, or launch from the exit area in the $x-t$ space of impulsive onedimensional flow. The tube flow within the plate is an $x-t$ centered expansion, while the diverging splash in the vacuum is a sequence of two-dimensional (axisymmetric) steady expansions. Figure 2 is a schematic of a two-dimensional steady expansion, fluid particle paths are shown as dotted lines.

\section{Splash flow}

The splash flow at a radial distance $R$ from the exit area and at time $t$ is related to the exit flow at $R=0$ by the constancy of mass flow between wave traversals, $\mathrm{w}=$ density $\cdot$ velocity $\cdot$ area, 


$$
\begin{aligned}
& \mathrm{W}_{\mathrm{i}(R, t)}=\left[\mathrm{F}_{1} \rho_{\mathrm{i}(R, t)}\right]\left(\mathrm{F}_{2} \mathrm{C}\right)\left(\pi \mathrm{r}_{0}^{2}\right)=\left[\rho_{0} \xi(R, t)\right](\mathrm{Gc})\left(2 \pi R^{2}\right) \\
& \mathrm{i}(R, t)=\operatorname{integer}\left(\frac{t-\frac{R}{\mathrm{GC}}}{\frac{\Delta \mathrm{L}}{\mathrm{C}}}\right) \geq 0 \\
& \mathrm{~F}_{1} \cdot \mathrm{F}_{2}=\left(\frac{2}{\gamma+1}\right) \frac{1}{\gamma-1} \cdot \sqrt{\frac{2}{\gamma+1}}=\sqrt{\left(\frac{2}{\gamma+1}\right)^{\frac{\gamma+1}{\gamma-1}}}=\mathrm{F} \\
& \mathrm{G}=\sqrt{\frac{2}{\gamma-1}} \\
& \zeta(R, t)=\frac{\rho_{\mathrm{i}(R, t)} \mathrm{r}_{0}^{2} \mathrm{~F}}{2 \rho_{0} R^{2} \mathrm{G}} \\
& R \geq \sqrt{\frac{\mathrm{r}_{0}^{2} \mathrm{~F}}{2 \mathrm{G}}}
\end{aligned}
$$

Here only the flow back toward the electron beam is considered. The mass flow at $R$ and $t$ corresponds to mass flow at $R=0$, with wave index $\mathrm{i}(R, t) . \mathrm{F}_{1},(0.6495)$, is the ratio of exit density at $\mathrm{M}=1$ to the exit density at stagnation conditions $(\mathrm{M}=0, \mathrm{i}=0,1,2, \ldots) . \mathrm{F}_{2},(0.8660)$, is the ratio of the speed of sound at $M=1$ to the speed of sound at stagnation temperature (the stagnation speed of sound is $\mathrm{C}$ ). F, $(0.5625)$, is the product of $F_{1}$ and $F_{2}$, and appears in the formula for the total mass flow out of the streamtube. Gc, $(1.7321 \cdot \mathrm{c})$, is the maximum speed fluid can attain $(\mathrm{M}=\infty)$. The maximum turning angle for $\gamma=5 / 3$ flow in accelerating from $\mathrm{M}=1$ to $\mathrm{M}=\infty$ is $90^{\circ} . \zeta(R, t)$ is a density ratio defined to be no larger than unity for $R$ below $\mathrm{r}_{0} \sqrt{ }[\mathrm{F} /(2 \mathrm{G})],\left(0.403 \cdot \mathrm{r}_{0}\right)$. The flow is assumed to move as an expanding hemisphere, this approximation is accurate for $R$ beyond a distance comparable to the exit diameter. For this model, the transition between one-dimensional flow at $\mathrm{M}=1$ and hemispherical flow at $\mathrm{M}=$ $\infty$ is designated to occur over a negligibly short distance $r_{0} \sqrt{ }[F /(2 G)]$ and time $\left[\mathrm{r}_{0} /(\mathrm{Gc})\right] \sqrt{ }[\mathrm{F} /(2 \mathrm{G})]$. 


\section{Examples}

Four examples described in the work cited in reference (1) were also calculated using the formulas presented in this report. The first three examples had an electron beam of $3.8 \mathrm{kA}$ at $5.25 \mathrm{MeV}$ within a diameter of $1 \mathrm{~mm}$ and a pulse width of $70 \mathrm{~ns}$. These examples each had a target of the following material and thickness: (1) aluminum at $0.8 \mathrm{~mm},(2)$ tantalum at $1.0 \mathrm{~mm}$ and, (3) copper at $0.8 \mathrm{~mm}$. Los Alamos experimentalists had measured the speeds of the splash flows for these three examples; calculations had been performed for examples (1) and (3). The fourth example corresponds to a projected electron beam facility at Los Alamos and is a purely computational exercise. This last case assumes an electron beam of $4.5 \mathrm{kA}$ at $20 \mathrm{MeV}$ within a diameter of $0.025 \mathrm{~mm}$ and a pulse width of $70 \mathrm{~ns}$. The target for this case was aluminum at $1.0 \mathrm{~mm}$ thickness. Table 1 shows a comparison between the results of the model in this report (columns three, four, five) and the Los Alamos work (last column).

\section{Table 1: Comparison of splash speed estimates}

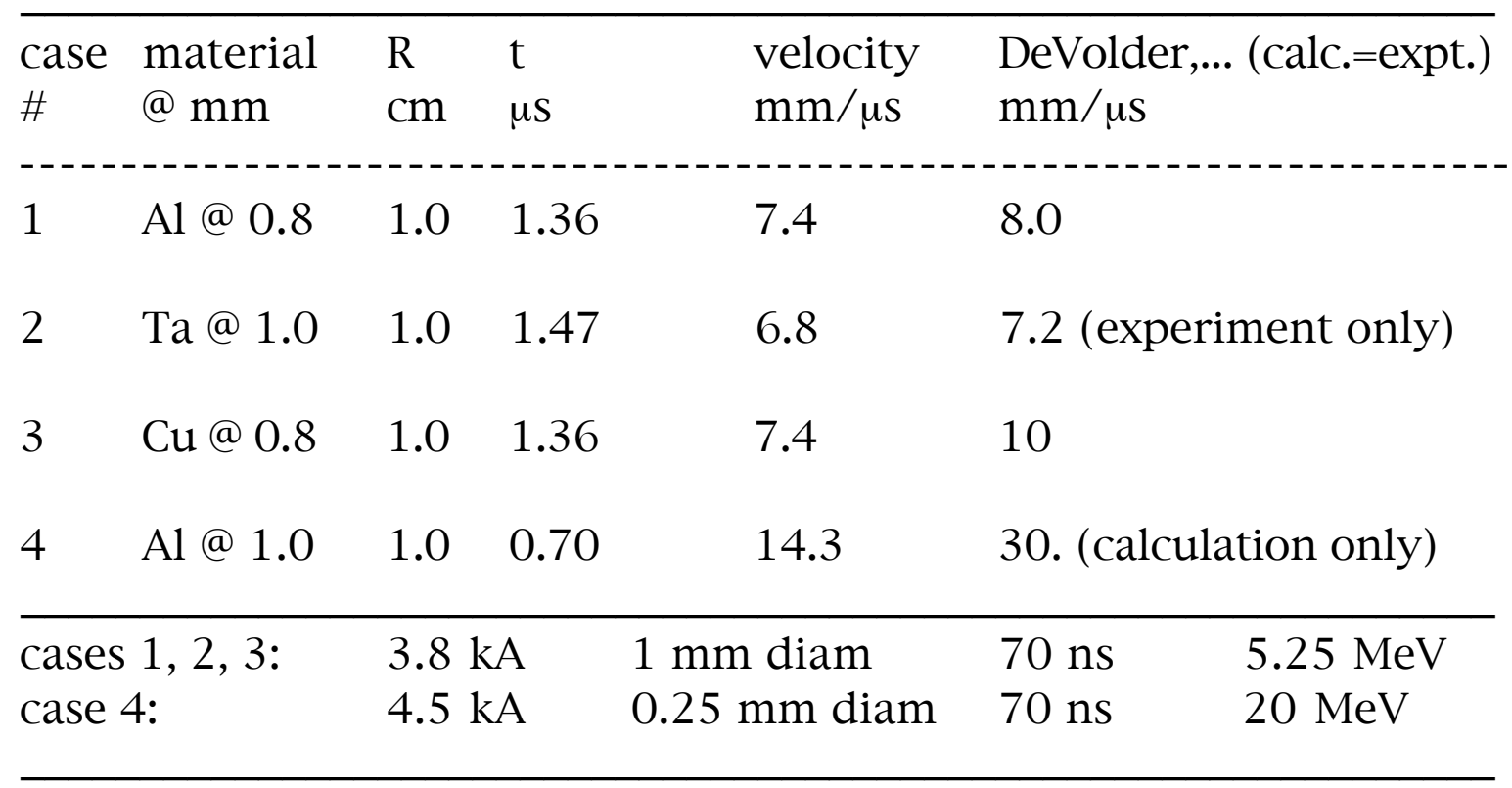

The analytical model agrees with the Los Alamos work because each method arrives at a similar stagnation temperature, which is the fundamental parameter of the problem, and the high density assures 
a flow at equilibrium. I calculate the stagnation temperature for each example as follows: (1) $3 \mathrm{eV}$, (2) $15.7 \mathrm{eV}$, (3) $6.9 \mathrm{eV}$, and (4) $11 \mathrm{eV}$.

I would like to present some detailed results from my calculation of example (1). Figure 3 shows the aluminum density ratio at the exit plane of the streamtube on the side of electron impact as a function of time in microseconds. The density decreases in a staircase fashion at intervals determined by the travel time of sound waves through the streamtube. The corners of this temporal staircase are rounded if waves of finite thickness are used during analysis in place of clumped waves. This density drops by three orders of magnitude in a microsecond, so at that time a metallic gas with a density of one and a half standard atmospheres flows out of the plate.

The splash flow for example (1) is illustrated in Figure 4, a three dimensional plot of $\xi(R, t)$. This flow moves $1.0 \mathrm{~cm}$ in $1.36 \mu \mathrm{s}$, for a speed of $7.4 \mathrm{~mm} / \mu \mathrm{s}$, and it has a stagnation temperature of nearly 3 $\mathrm{eV}$. The "exclusion zone" (subscript "E") for this example is also noted in Figure 4. The relative density ahead of the material front was set equal to the lowest relative density in the domain of the calculations $\left(<10^{-6}\right)$ to avoid the problem of plotting $\log (0)$. This plot shows the combined effects of the decrease of source density over time and the attenuation due to the expansion in space.

A sequence is shown in Figure 5 of radial profiles of normalized density for example (1). In this particular case the venting is so rapid and the source volume so small that the mass of the source disappears and the splash is an expanding shell. This feature is evident in the Los Alamos calculations of example (1), see reference (1). This shell flow also occurs in examples (2) and (3), but not in example (4), where the expansion is uniform. For examples (1), (2), and (3), the material shells have a relative density of about $5 \times 10^{-4}$ and an extent of about $2.0 \mathrm{~mm}$ when the splash reaches $1.0 \mathrm{~cm}$. Example (4) has a fairly uniform relative density, being about $5 \times 10^{-5}$ when the splash reaches $1.0 \mathrm{~cm}$ at $0.7 \mu \mathrm{s}$.

These examples show that metallic clouds of between 0.1 and 1.0 standard atmospheric density will extend out to $1.0 \mathrm{~cm}$ from millimeter-thick target plates by $1.0 \mu$ s after impact by electron beams of the type described. 


\section{Possible future work}

Does the splash matter? Will a splash affect a subsequent pulse as assumed? One exercise to explore this question is to find the integral

$$
\Delta E(x, t)=\int_{-\infty}^{x}\left[1.6 \frac{\mathrm{MeV} \cdot \mathrm{cm}^{2}}{\mathrm{gm}}\right] \rho_{0} \xi(x, t) d x
$$

This is the energy deposited by a single electron by position $x$ along the direction of the electron beam. It may be that for a few pulses most of the energy is still deposited in the plate and little is lost in the splash flows. On the other hand, subsequent pulses may heat and ionize the splash flows, creating dense plasmas that can interact in more elaborate ways with later pulses. Quantifying these conjectures is much easier given the splash density distribution $\rho_{0} \xi(R, t)$ and the average density of the streamtube $\rho_{i}$. Another investigation is to estimate the variation with $x$ of the electron multiple scattering angle. This scattering angle is a statistical average of electron deflections and determines the extent of lateral beam diffusion while penetrating matter, see Fermi (2). It may be that the line integral of density through the splash is insignificant in comparison to that through the fluidized plate.

Another exercise is to design a target with teeth (like the rack in a gear mechanism) and lateral motion so that splashes are confined within trenches that are swept away. A lateral motion of $1.0 \mathrm{~mm} / \mu \mathrm{s}$ would allow for a $1.0 \mathrm{~mm}$ trench (say $5.0 \mathrm{~mm}$ deep) and $1.0 \mathrm{~mm}$ ridge to sweep by a $1.0 \mathrm{~mm}$ diameter beam "spot" every $2.0 \mu \mathrm{s}$. This speed is comparable to that of a high velocity bullet or the tip speed of a 1.0 meter turbine at 20,000 rpm. These may seem like extreme solutions to the problem, however they may be more realistic and reliable than the alternative of pulsed magnetic steering of multi-MeV beams between pulses separated by one or two microseconds.

Perhaps the most promising exercise is to explore the parameter space offered by a variety of electron beams and targets, and then suggest that the most interesting cases be subjected to comprehensive computation by groups like DeVolder's at Los Alamos. If further 
examples prove that my energy deposition model is widely applicable then a significant amount of computational work can be eliminated.

\section{Postscript}

During the time this report was being reviewed and prepared for distribution work continued. The model has been expanded to include the effects of energy-dependent deposition, so that $(1 / \rho) \mathrm{dE} / \mathrm{dx}$ $=1.6 \mathrm{MeV} \cdot \mathrm{cm}^{2} / \mathrm{gm}$ is now determined by the Bethe formula as presented in reference (3). This energy deposition results from the loss of beam energy by collisional ionization of the target. Beam energy is also lost by bremsstrahlung radiation. This radiative term is smaller than the collisional term, and is lost to vacuum from thin targets. No significant effect is observed in the flows calculated with this more detailed energy deposition formalism. The values of $(1 / \rho) \mathrm{dE} / \mathrm{dx}$ still hover within $20 \%$ of $1.6 \mathrm{MeV} \cdot \mathrm{cm}^{2} / \mathrm{gm}$. These and other details will be investigated further.

Another recent addition is a model of nonequilibrium plasma flow. A small portion of the original ionization remains "frozen" in the cold expanding flow. For example (1), described earlier, this plasma has a density of about $0.6 \%$ of the neutral density at the front of the flow, and an electron temperature of $0.2 \mathrm{eV}$ there. This is consistent with the results presented in reference (1). A report on this new work is being written.

\section{Acknowledgments}

I am very grateful to Barbara DeVolder for sending me a copy of her poster paper, and for taking the time to explain it in detail during the ICOPS conference in May of this year. 


\section{References}

(1) B. T. DeVolder, T. J. T. Kwan, R. D. Fulton, D. C. Moir, D. M. Oro, D. S. Prono, "Computational and experimental studies of the beamtarget interaction for high-dose, multi-pulse radiography," Los Alamos National Laboratory, LA-UR-97-8, and the 24th IEEE International Conference on Plasma Science, 7P26, May 19-22, 1997, San Diego, CA.

(2) E. Fermi, Nuclear Physics, Revised Ed., University of Chicago Press, 1974.

(3) M. J. Berger, S. M. Seltzer, "Studies in penetration of charged particles in matter," National Bureau of Standards Nuclear Science Series Report No. 39, Washington DC: National Academy of Sciences-National Research Council Publication 1133, 1964.

(4) A. H. Shapiro, The Dynamics and Thermodynamics of Compressible Flow, Volume 1, New York: Ronald Press Company, 1953. 


\section{Figure legends}

(1) Clumped and finite waves. $x$-t schematics of streamtubes open at both ends with clumped (top) and finite-thickness (bottom) expansion waves. These waves move at the speed of sound, which varies locally as the temperature changes. Fluid particle paths are shown as dotted lines. Regions of static fluid have vertical particle paths, and regions of moving fluid have slanted particle paths. The expansion of the fluid appears as an increase in the separation of adjacent particle paths over time. Conditions at the exits are steady between the times waves arrive. The density at the exits is a staircase in time with intervals set by the traversal time of sound waves through the streamtube.

(2) Exit flow into vacuum. $x-y$ schematic of steady splash flow in the vacuum outside the plate. Expansion fans emanate from the lip of the exit area, and much of the flowfield is a region of interpenetrating expansion wavelets (for axisymmetric flow an expansion fan ring emanates from lip of the exit). Fluid particle paths are shown as dotted lines. Stipple patterns indicate regions within both, one, or none of the expansion fans. The mass flowing per unit time across a hemisphere at distance $R$ from the streamtube exit must equal the mass that flowed per unit time out of this end of the streamtube at a time earlier by the travel interval across $R$. The density at each point $(R, t)$ has its temporal dependence set mainly by the time dependence of the streamtube exit density, and its spatial dependence set by the two-dimensional expansion of the splash flow.

(3) Aluminum density ratio at exit plane. The history of the relative density at the exit of the streamtube for example (1) is a staircase with intervals set by wave travel times along the streamtube. Here the density drops three orders of magnitude in one microsecond. The sharp edges of this history are smoothed if finite thickness waves are used in the $\mathrm{x}-\mathrm{t}$ analysis of the streamtube flow. The rate at which this relative density drops depends on both the stagnation temperature of the fluid and the size of the reservoir, small hot volumes vent more quickly. 
(4) Aluminum splash at $7.4 \mathrm{~mm} / \mu \mathrm{s}$. The relative density of the splash flow for example (1) at distance $R$ and time $t$ over a domain of $1.0 \mathrm{~cm}$ and $1.36 \mu \mathrm{s}$. The "front" of the splash is visible as the large diagonal cliff with a slope of $7.4 \mathrm{~mm} / \mu \mathrm{s}$. The streamtube exit density history is seen at the $R=0$ plane, and radial profiles of splash density are seen as any snapshot $(t=$ constant) plane. Notice the shell-like structure of the splash flow at $t=1.36 \mu \mathrm{s}$. The relative density ahead of the front is set arbitrarily to avoid plotting $\log (0)$.

(5) Aluminum splash, density ratio @ radius (cm) @ time (s). A sequence of splash flow relative density profiles for example (1). Time $t$ is in seconds, distance $R$ is in centimeters, and relative density $\zeta$ is set to unity below distance $\mathrm{x}_{\mathrm{E}}$. The shell-like nature of the flow is clearly visible. Sharp edges in the profiles are rounded if finite thickness waves are used in place of clumped waves. The shell is about $2.0 \mathrm{~mm}$ thick when the front has reached $1.0 \mathrm{~cm}$ at $1.36 \mu \mathrm{s}$. The density of this shell is about one standard atmosphere, or $2.7 \times 10^{19}$ aluminum atoms per cubic centimeter. 
Figure 1: Clumped and finite waves
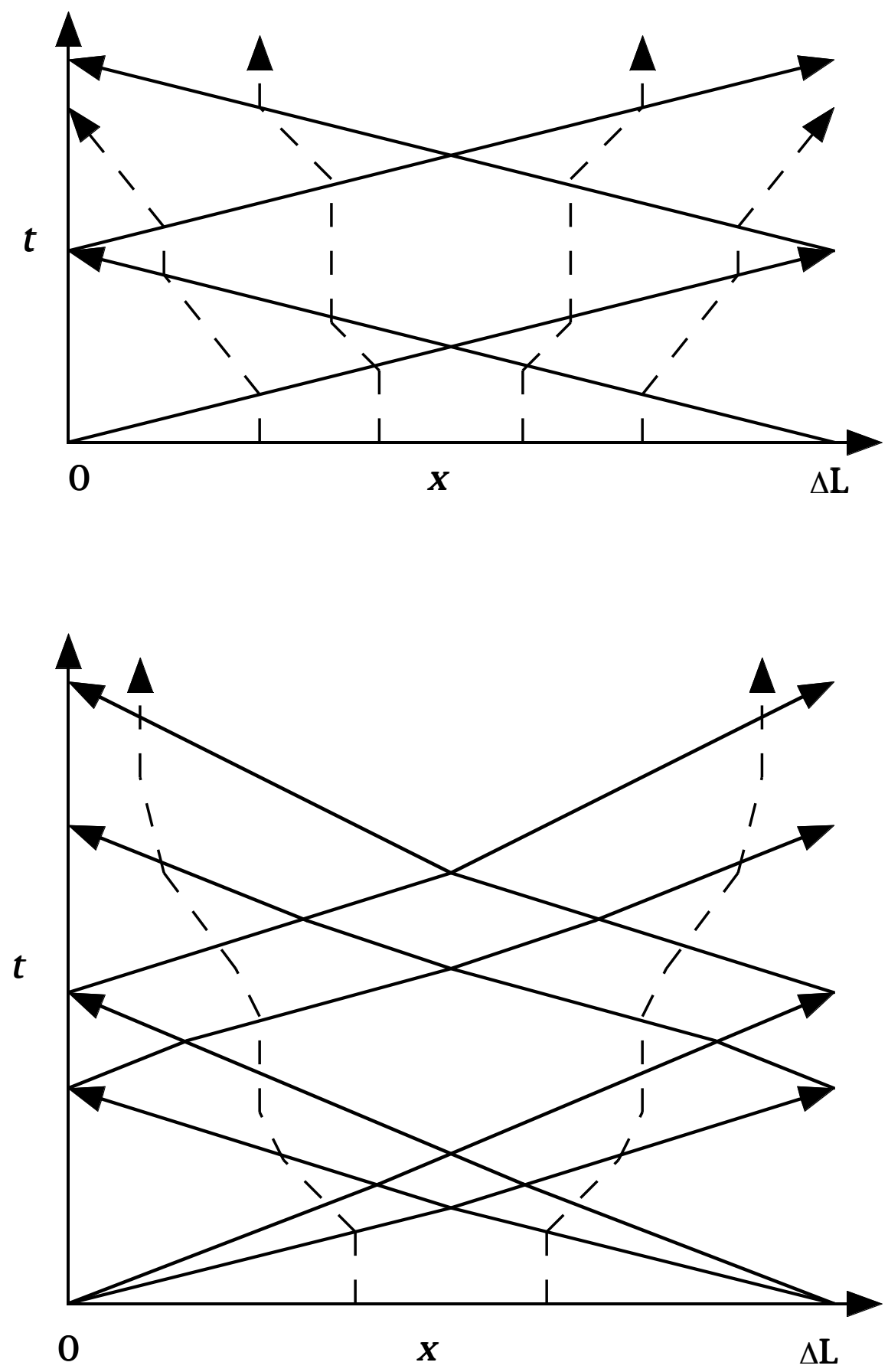
Figure 2: Exit flow into vacuum

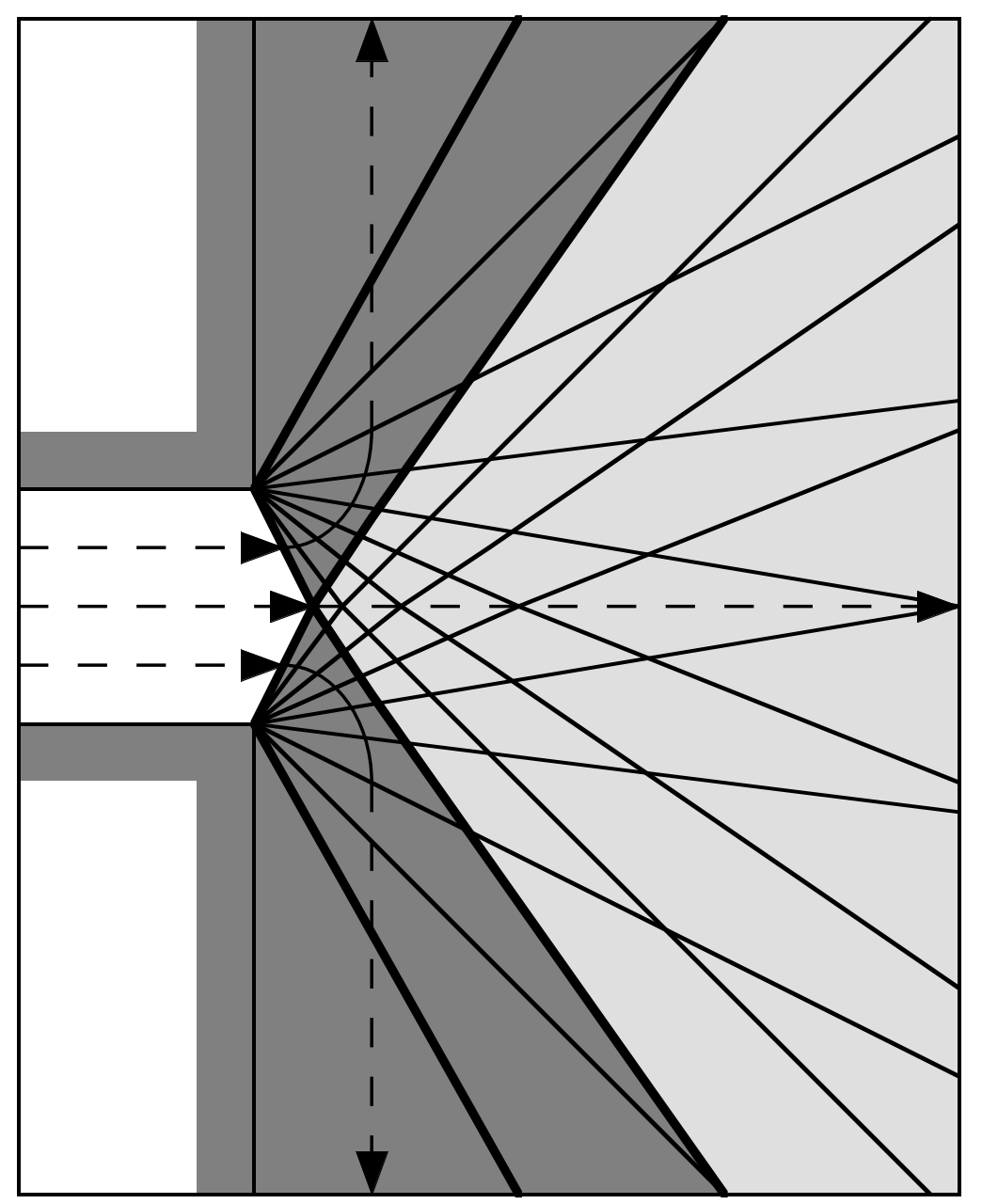


Figure 3: Aluminum density ratio at exit plane

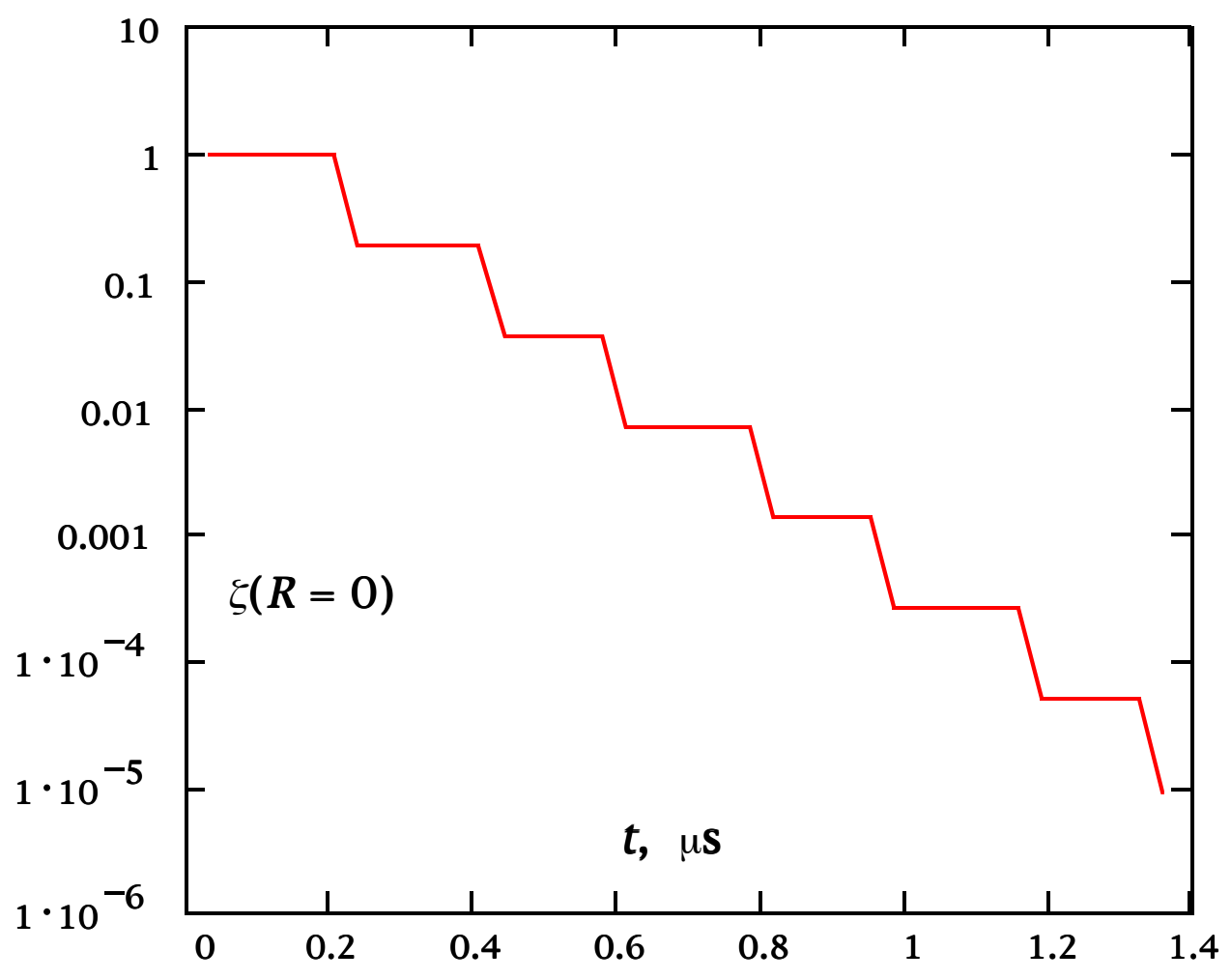


Figure 4: Aluminum splash at $7.4 \mathrm{~mm} / \mu \mathrm{s}$

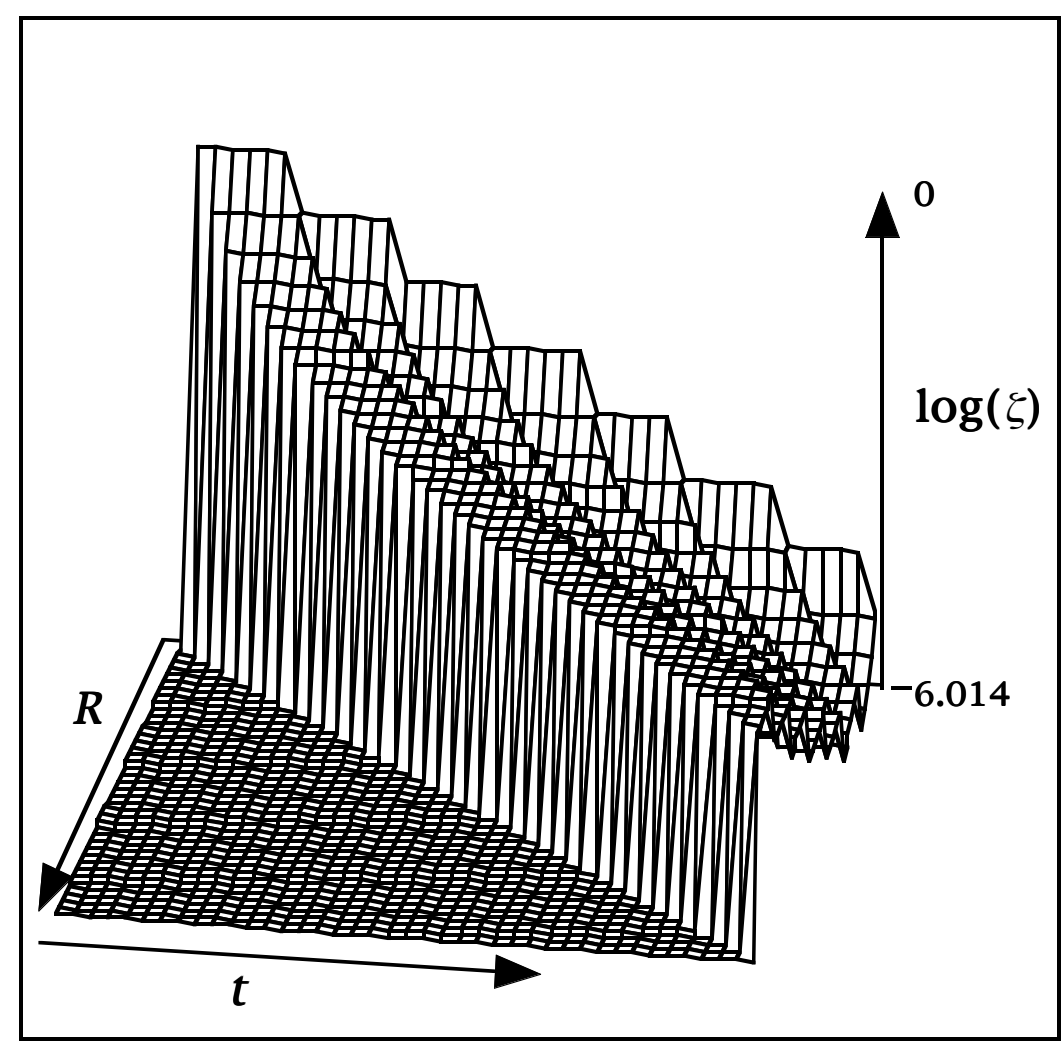

$\begin{array}{ll}\text { Energy }=5.25 & \mathrm{MeV} \\ \text { Temp }=2.985 & \mathrm{eV} \\ \text { Radius }=1 & \text { centimeters } \\ \text { Time }=1.36 \cdot 10^{-6} & \text { seconds }\end{array}$

Beyond (s, cm):

$$
\begin{aligned}
& \mathrm{t}_{\mathrm{E}}=2.766 \cdot 10^{-8} \\
& \mathrm{x}_{\mathrm{E}}=0.02
\end{aligned}
$$


Figure 5:

Aluminum splash, density ratio @ radius $(\mathrm{cm}) @$ time $(\mathrm{s})$ [after $\mathrm{t}_{\mathrm{E}} \&$ beyond $\mathrm{x}_{\mathrm{E}}$ ]
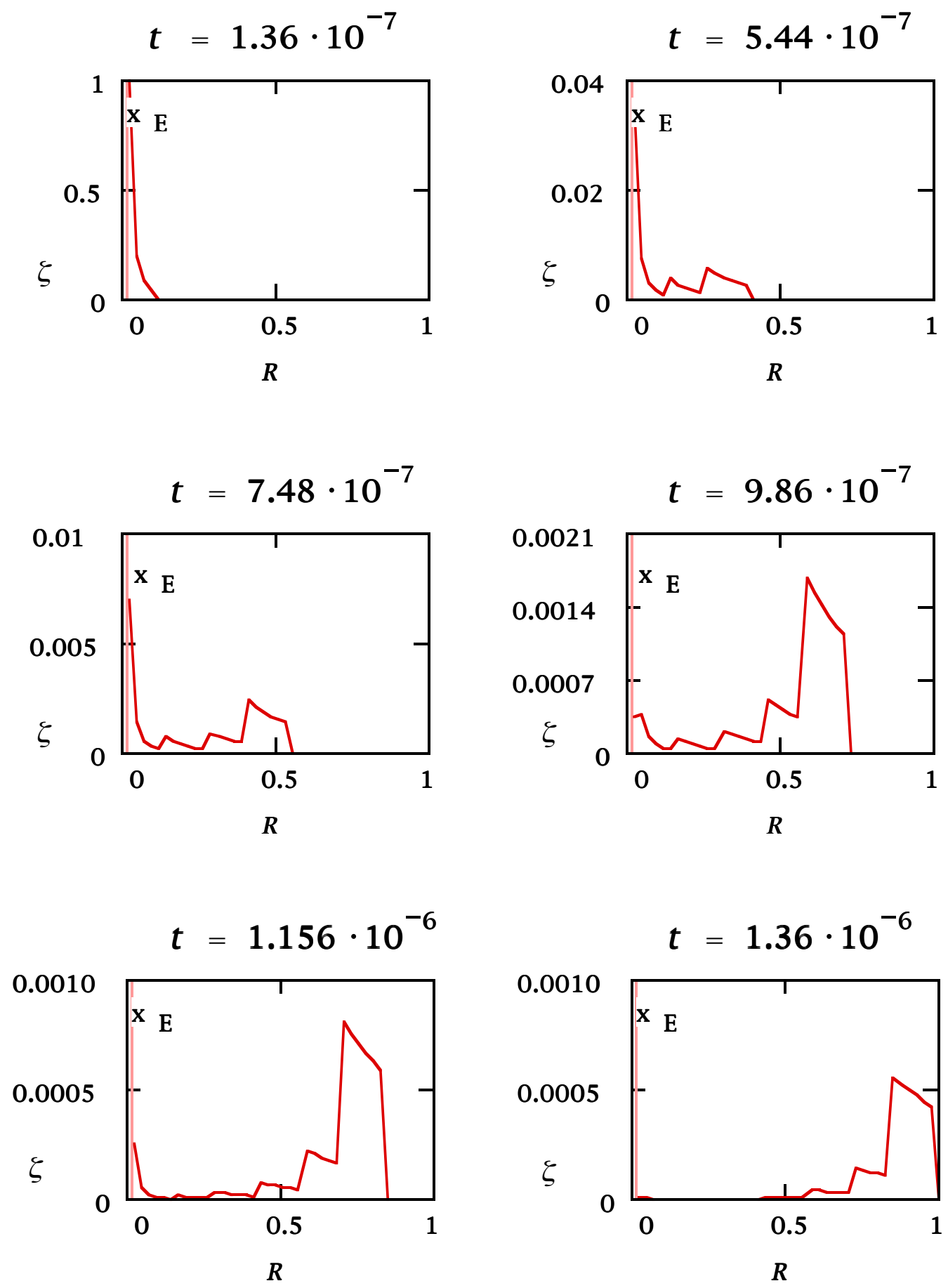


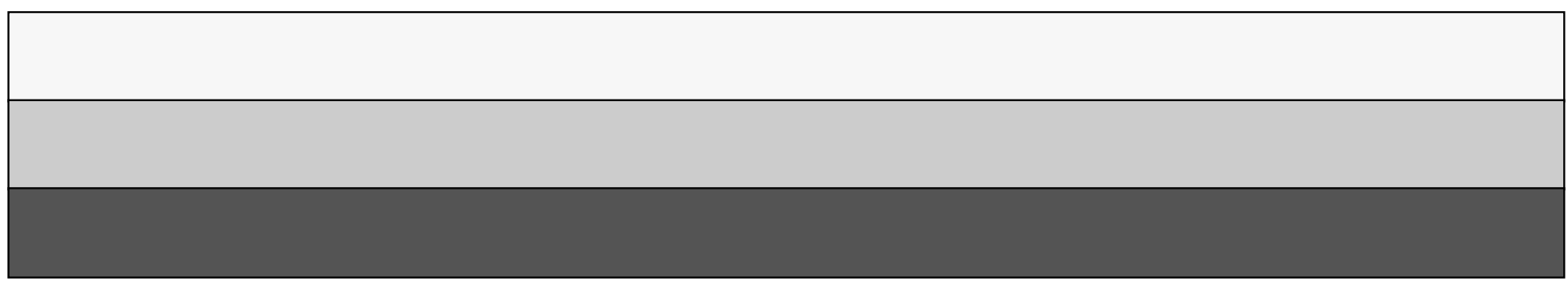

